\title{
La sustentabilidad de Internet de las Cosas
}

\section{Zito, Maximiliano [ver currículum del autor, docente de la Facultad de Diseño y Comunicación]}

Resumen: El término Internet de las Cosas ha permeado nuestra sociedad en los últimos años de manera especialmente intensa en los medios de comunicación. Si bien el término no es nuevo, la masificación de dispositivos electrónicos, la posibilidad real de analizar grandes cantidades de datos a través del Big Data y de extraer conclusiones útiles de ellos parece haber abierto definitivamente la puerta para una explosión del loT.

El presente artículo busca reflexionar acerca de un problema que no parece tratarse de manera adecuada: sus implicancias ambientales y sociales. En definitiva, en su sustentabilidad. En un mundo que está alcanzando sus límites, ¿es posible que Internet de las

Cuadernos del Centro de Estudios de Diseño y Comunicación №70

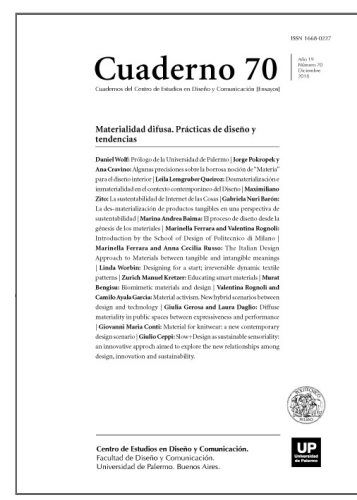

ISSN: 1668-0227

Materialidad difusa.

Prácticas de diseño y

tendencias

Año XIX, Diciembre 2018, Buenos Aires, Argentina | 172 páginas

descargar PDF

ver índice de la publicación

Ver todos los libros de la publicación

compartir en Facebook Cosas pueda desarrollarse? y en caso de hacerlo, ¿será para todos, ayudando a reducir la brecha entre los que más tienen y los que menos poseen? o por el contrario, ¿aumentará dichas diferencias?

Palabras clave: Internet de las Cosas - loT - Sustentabilidad - Impactos ambientales - Paradoja de Jevons Límites del crecimiento.

$\left(^{*}\right)$ Investigador especializado en al área de Diseño para la Sustentabilidad, Centro INTIDiseño Industrial. Diseñador Industrial, Facultad de Arquitectura Diseño y Urbanismo (FADU), Universidad de Buenos Aires (UBA). Especialista en Gestión Ambiental de Producto (ISM-España), Maestría en gestión de la Energía (Universidad de Lanús), Especialista en Gestión Estratégica de Diseño (FADU-UBA).

El término Internet de las Cosas, también denominado con sus siglas en inglés loT (Internet of Things) ha permeado nuestra sociedad en los últimos años de manera especialmente intensa en los medios de comunicación. Si bien el término no es nuevo, también es cierto que las capacidades tecnológicas para implementarlo de manera más efectiva son más recientes. La masificación de dispositivos electrónicos, la 
posibilidad real de analizar grandes cantidades de datos a través del Big Data y de extraer conclusiones útiles de ellos parecen haber abierto definitivamente la puerta para una explosión del loT.

El término loT está en desarrollo y por ende aún no tiene una definición aceptada universalmente. Una encuesta realizada a varios investigadores en 2013 refleja esta situación1. Sin embargo, se podría definirlo como un concepto que se refiere a la interconexión digital de objetos cotidianos con internet2. Con objetos cotidianos se hace referencia a todo tipo de productos como ser heladeras, licuadoras, automóviles, iluminación y en general cualquier aparato que interactúe de alguna forma con una persona.

Otro término que se comienza a escuchar es Internet de Todo, como se lo conoce por sus siglas en Inglés loE (Internet of Everything). En este caso, se hace referencia a la conexión inteligente no sólo de las cosas, sino también de las personas, procesos y datos. Es decir que a loT se la agregaría la inteligencia necesaria para que todo el sistema pueda funcionar de una manera convergente y orquestada.

Ahora bien, más allá de las definiciones, lo cierto es que si actualmente se busca información de estos conceptos, rápidamente se pueden encontrar numerosos artículos y notas cuyos contenidos podría agruparse en dos tópicos. Por un lado las bondades que traerá poder controlar todos estos dispositivos y como ello facilitará la vida cotidiana; y por el otro, los peligros implícitos de tanto control. Estos últimos básicamente agrupados en torno a la pérdida de privacidad y a la posibilidad de que un hacker se haga del control de nuestros objetos, y por extensión de nuestra vida.

El presente artículo no pretende ahondar sobre estos temas. No porque carezcan de importancia, sino mas bien porque ya hay mucho escrito, y por personas más entendidas del tema. Aquí más bien se busca reflexionar acerca de otro problema relacionado a Internet de las Cosas que no parece tratarse de manera adecuada: sus implicancias ambientales y sociales. En definitiva, en la sustentabilidad de loT.

El mundo, como se sabe, está alcanzando sus límites en la disponibilidad de varios de sus recursos físicos. Esa situación se encuentra bien documentada por parte de numerosos estudios de conocidos organismos como la Organización para la Cooperación y el Desarrollo Económicos (OCDE)3 y del sector privado como la consultora privada KPMG4 , por mencionar solo algunos. El mecanismo de crecimiento desmedido que luego se frena abruptamente al encontrarse con los límites que le impone su entorno en realidad se puede aplicar a muchos otros órdenes de la vida y comúnmente se lo conoce como Crecimiento Exponencial. En la ecología por ejemplo, se aplica a cómo una población de una determinada especie puede crecer exponencialmente (si tiene las condiciones propicias en su ambiente), hasta llegar al límite de sus recursos. En este punto el crecimiento se frena y luego sobreviene una fuerte caída en la cantidad de individuos.

La loT, como tecnología emergente, debería tener en cuenta estas limitaciones a la hora de plantear su crecimiento. De igual manera que una especie de seres vivos lo hace con los recursos disponibles en el ambiente.

Veamos algunos datos para considerar esta cuestión.

En una conferencia sobre el tema5, se pudo observar que las tendencias indican que para el año 2020 habrá entre 17 y 30 billones (si, billones) de objetos conectados entre sí. Y por supuesto, detrás de ellos, la 
descomunal cantidad de datos que se compartirán. Los mismos estudios los estiman en 4.3 ZB anuales. ¿Cuánto es esto? Para entender estas magnitudes se deben hacer algunos cálculos. Por ejemplo, cuando comenzó la era de Internet entre 1990 y 1995, se calcula que se utilizaban en todo el mundo 204 Terabyte (TB) al mes. Esto equivale a 200 computadoras Imac como la que están en cualquier oficina de diseño grá- fico en la actualidad. Continuando en el tiempo, de 2005 a 2010 aumentó dicho consumo a unos 20 mil Petabyte (PB) al mes. Es decir a la capacidad de almacenamiento de más de 20 millones de Imac. Y si se llega a los mencionados 4.3 Zettabyte (ZB), serían unas 4600 millones de computadoras de la manzanita. Para dimensionar mejor este número, se trata de más de la mitad de la población mundial. Y no se continúa del 2020.

La pregunta que surge luego es si toda esta capacidad colosal de almacenamiento es gratuita para nuestro planeta. No ya en términos monetarios, que son relativos, sino en términos de recursos físicos. Se puede estar seguro que no. Cada Megabyte de almacenamiento tiene un costo ambiental. Utiliza materiales físicos, se mueve desde el sitio de fabricación al de uso, emplea energía para poder utilizarse por internet y para ser refrigerado, y por último, al finalizar su vida útil debe ser reprocesado (en el mejor de los casos).

¿Cuál es el costo ambiental de extraer los minerales, transformarlos en materiales y utilizar ese almacenamiento? Para realizar estos cálculos se suele utilizar la metodología de Análisis de Ciclo de Vida, la cual contempla cuantificar el impacto ambiental de un producto a lo largo de cinco etapas: extracción, fabricación, uso, distribución y fin de vida.

Además, hasta ahora se mencionó el costo ambiental de crear y utilizar el almacenamiento. Para realizar la cuenta completa, habría que sumarle este mismo costo ambiental de producir todos los nuevos objetos inteligentes. Porque seguramente, no se podrá utilizar la misma licuadora, o los mismos anteojos, o el mismo automóvil que existen hoy día. Es decir, hay que reconvertir toda la población mundial de objetos. Claro que desde un punto de vista económico, este cambio ofrece inmensas oportunidades de negocios. Pero se insiste en la necesidad de reflexionar sobre los impactos ambientales y sociales de esta enorme transformación que se avecina.

Al igual que una moneda que tiene dos caras, loT tiene un lado ambiental positivo. Tanto control de aparatos permitirá poder regular mejor el uso de energía, del agua y de otros muchos consumibles. Las computadoras podrán decidir de manera más racional y eficiente que las personas cuando bajar la temperatura de un aire acondicionado, cuando levantar una persiana para que ingrese más luz natural o cuando apagar una luz en un cuarto que no hay nadie. También podrá conducir un automóvil de manera más eficiente, racionalizando el consumo de combustible.

Muchos de los productos que requieren consumibles en su utilización (como agua, energía, aceites y otros), presentan sus mayores impactos ambientales en la etapa de uso. Esto no es mera teoría, ya que mediante la misma metodología mencionada anteriormente del Análisis de Ciclo de Vida se ha verificado esta premisa en numerosos aparatos. Aires acondicionados, lavarropas, heladeras, cafeteras y un sin fin de productos similares entran dentro de este grupo. En este sentido, la loT puede realizar aportes muy significativos. En primer lugar, el poder recopilar en gran escala datos acerca de las conductas de consumo por parte del público abre la puerta a tener la posibilidad de generar políticas de ahorro y eficiencia basadas en datos contrastados. Asimismo, la verificación del cumplimiento de dichas políticas también será una realidad alcanzable. Es evidente que esto abre oportunidades para mejoras de eficiencia enormes, lo cual podría traducirse en significativos ahorros. 
Pero como en esta vida no siempre todo lo que reluce es oro, las mencionadas mejoras en la eficiencia no necesariamente podrían conducir a un ahorro global en los recursos. Ejemplos de ello hay muchos, como ser los motores de automóviles. Comparados con los motores de hace 15 o 20 años, los actuales son más chicos y consumen menos combustible por kilómetro recorrido, es decir que se puede afirmar que en promedio, cada automóvil hoy es más eficiente que uno de hace 20 años. Sin embargo, la cantidad global de vehículos ha crecido de forma exponencial en el mundo, con lo cual el ahorro a nivel global no existe. Es más, hoy se consume mayor cantidad de combustible que hace 20 años.

Esta situación, donde mejoras de eficiencia individuales no conducen a los supuestos ahorros globales en realidad ya fue observada en el siglo XVIII. En aquel momento un economista inglés de nombre Jevons, en plena Revolución Industrial fue encomendado para analizar y estimar la duración de las reservas de carbón en Inglaterra. El carbón era estratégico porque en aquel entonces era la fuente energética principal para alimentar a las máquinas de vapor.

Lo que Jevons descubrió es que a medida que las máquinas mejoraban su eficiencia individual, éstas eran utilizadas en mayor cantidad de tareas. Por consiguiente, a pesar de que cada máquina consumía menos carbón para realizar la misma actividad, a nivel global se utilizaban cada vez más máquinas y el consumo general de carbón crecía.

Esta paradoja, donde a pesar de mejorar la eficiencia a nivel individual se obtiene como resultado un mayor consumo global se la llamó Paradoja de Jevons.

Redefiniendo el confort

Como ya se explicó, uno de los aspectos más promocionados de la Internet de las Cosas es la mejora que provocará en el confort de los usuarios. Para ello se suelen dar argumentos acerca de lo fácil que será que una heladera nos informe que falta leche, o que se acabó tal o cual queso que siempre consumimos. Pero claro, en el mundo real nada es "gratis". Y este servicio, tiene un costo ambiental. Aparece entonces la dicotomía entre ambiente y confort. ¿Qué se debe priorizar? ¿La comodidad de que la heladera me recuerde que falta leche, o más aún que mande a comprarla en mi lugar? o por el contrario, ¿El cuidado del ambiente, en cuyo caso funciones como la mencionada no deberían tener lugar?

En este punto llegamos a la espinosa cuestión de precisar que entendemos por confort. La Real Academia Española define al término como "Bienestar con comodidad material", aunque por supuesto que esta definición tiene un alto grado de subjetividad.

Entender este término de manera adecuada es crucial para los diseñadores, ya que en definitiva es el usuario del producto quién tiene la última palabra. Y es claro que un producto que ofrece mayor confort, será más valorado por el usuario.

Pero, ¿hasta dónde avanzar en la búsqueda de más confort? 
Muchos de los objetos que hoy nos proporcionan esta cualidad -y a los que no estamos dispuestos a renunciar de manera voluntaria- hasta hace unas décadas atrás apenas se consideraban necesarios. Si se le pregunta a la mayoría de las personas que hoy tienen 60 o 70 años, seguramente contestarán que de jóvenes vivían en condiciones más austeras y no por ello consideraban que vivían sin confort. Es decir, que el confort es subjetivo, según la época y el contexto.

Contexto mundial, ¿alcanzando los límites?

Como ya se mencionó, para que la Internet de las Cosas se pueda implementar y masificar, es necesario una enorme transformación de los productos - tanto que usan los usuarios como los que sirven de soporte para que funcionen éstos-, lo cual probablemente exigirá una gasto fenomenal de capital natural. Llamamos a esto todas las materias primas y la energía necesaria para transformarlas en los productos requeridos. Sin contar por supuesto con la energía que consumirá toda esta gigantesca red de aparatos interconectados de manera permanente. Estos aspectos espinosos se suelen pasar por alto.

La electrónica de precisión, los láseres, las turbinas de energía eólica y muchos otros aparatos de nueva tecnología necesitan de muchos minerales específicos para funcionar. El Servicio Geológico de los Estados Unidos (USGS por sus siglas en inglés) tiene bajo seguimiento las reservas y consumo global de 58 Recursos Naturales No Renovables (RNNR) -minerales, energía y metales-, y según información de la agencia muchos de éstos no tienen reservas para seguir incrementado su extracción de manera significativa. Una situación similar se da con las llamadas Tierras Raras6. Neodimio, Cerio y Lantano son algunos de estos elementos sumamente necesarios para la tecnología actual. China posee prácticamente el monopolio de su producción7 ,y a partir del 2010 viene aplicando restricciones a su exportación. Todo esto sugiere que el mundo no puede contar con la cantidad y a un precio asequible lo que necesita, y esto sucede hoy, con una demanda mucho menor de la previsible. Recordando que el término Sustentabilidad (o Sostenibilidad)8 refiere no solamente a lo ambiental sino también a lo social y económico, se hace necesario mencionar estos dos aspectos adicionales para analizar la sustentabilidad de Internet de las Cosas.

En este sentido es importante entender la tendencia que siguen los precios de algunos de los minerales y metales para estimar como se comportarían en un previsible escenario futuro de mayor demanda.

Jeremy Grantham, cofundador de GMO -una de las mayores gestoras de activos del mundo-, sorprendió a todos cuando publicó un impactante informe9 en el que diseccionaba meticulosamente la situación de los recursos no renovables del mundo. Ya en el inicio del informe, se hace eco de un dato demoledor: en tan solo 8 años se han revertido 100 años de tendencias a la baja en los precios de las materias primas. Para ello utiliza un Índice de commodities GMO, el cual sintetiza el comportamiento de 33 materias primas, y donde se puede observar el incremento que vienen sufriendo como tendencia a mediano plazo. Por lo tanto, un escenario donde la mayor demanda de materias primas se golpeará con límites físicos, cabe preguntarse si la masificación de la loT será una realidad, ó solo quedará restringida a un grupo reducido de la población mundial, ampliando la brecha de desigualdad actual.

Reflexión final 
Quizá para el público general el concepto de la Nube evoca algo etéreo, intangible. Pero se puede estar seguro que atrás de cada megabyte que circula existen elementos muy físicos que deben existir, y que deben convivir en una estructura sumamente compleja para que funcionen como se espera.

Es necesario reflexionar sobre el confort, aspecto espinoso y directamente relacionado. Hasta ahora, se puede decir que el ser humano en término generales ha ido subiendo su umbral de confort. Por supuesto que hay enormes cantidades de personas en el mundo que están lejos de dicho umbral, pero podemos decir que en términos generales el ser humano a logrado aumentarlo. También es cierto, que mucho de ese confort está relacionado a poseer determinados objetos físicos. En todo caso, lo que es indudable es que el aumento de confort trajo aparejado un incremento en el uso de distintos recursos naturales. En definitiva, un mayor impacto ambiental.

Hoy se está llegando a la encrucijada de haber definido un nivel de confort muy alto -en términos ambientales-, el cual debido a una incipiente pero creciente restricción de recursos naturales, cada vez se revela más difícil de alcanzar para toda la humanidad. La Huella Ecológica10 es un indicador que claramente muestra esta situación, donde para compensar el consumo actual global se necesitan 1,4 mundos. Y este resultado es un promedio, donde un importante sector de la población mundial está marginado de los parámetros de consumo -y confortde los países desarrollados. Si todos vivieran según éstos, se estiman que se requerirían 4 planetas tierras para sostenerlo.

Internet de las Cosas, como está promocionado hoy en día, apunta sin duda a elevar aún más el estándar de confort. ¿Seremos capaces de utilizarla como herramienta para mejorar el ambiente y la sociedad, incorporando a los excluidos de hoy y de mañana? O por el contrario, nuestra insistencia en quererlo todo, en un mundo finito, provocará que loT sea utilizada por una reducida minoría de la población, incrementando el impacto sobre nuestro planeta y aumentado la brecha entre los afortunados y los desposeídos.

En definitiva, sólo el tiempo dirá la forma de implementación de la loT. Lo que sí es seguro es que para que ésta pueda crecer como anuncian los gurúes del tema, será necesario que continúe vigente el modelo actual. Es el escenario que en las simulaciones se lo suele denominar Business as Usual (expresión que podría traducirse en Negocios como Siempre). Es el modelo vigente. Es, en definitiva, el modelo que hasta ahora ha logrado burlar los límites de la física. ¿Por cuánto tiempo logrará hacerlo?

Notas

1. Charith Perera, Arkady Zaslavsky, Peter Christen, and Dimitrios Georgakopoulos (2013). «Context Aware Computing for The Internet of Things: A Survey». Communications Surveys Tutorials, IEEE. Early Access (n/a): 1-44. doi:10.1109/SURV.2013.042313.00197.

2. Conner, Margery (27 de mayo de 2010). Sensors empower the "Internet of Things" (Issue 10). pp. 32-38. ISSN 0012-7515.

3. OECD Environmental Outlook to 2050: The Consequences of Inaction -ISBN 978-92- 64-122161 (C) OECD 2012.

4. Informe Future State 2030: The global megatrends shaping governments, pág. 43 (2014 KPMG). 
5. Fábricas Inteligentes: Sistemas Ciberfísicos para la Producción. Ing. Victorio Bentivogli, ADIMRA (30/08/2016).

6. Tierras Raras es el nombre común de 17 elementos químicos: escandio, itrio y los 15 elementos del grupo de los lantánidos (lantano, cerio, praseodimio, neodimio, prometio, samario, europio, gadolinio, terbio, disprosio, holmio, erbio, tulio, iterbio y lutecio).

7. Concern grows over China's dominance of rare-earth metals, Physics Today (mayo 2010). http://scitation.aip.org/content/aip/magazine/physicstoday/article/63/5/10.1063/1. 3431322

8. Se define "el desarrollo sostenible como la satisfacción de «las necesidades de la generación presente sin comprometer la capacidad de las generaciones futuras para satisfacer sus propias necesidades". (Informe titulado "Nuestro futuro común" de 1987, Comisión Mundial sobre el Medio Ambiente y el Desarrollo).

9. Viviendo en un planeta finito, reporte Estado del Liderazgo en la Sustentabilidad (Universidad de Cambridge, diciembre 2012)

10. Desarrollada por la organización Global Footprint Network. Es un indicador que contabiliza la cantidad de territorio necesario para producir los recursos y asimilar los residuos de una población específica por un tiempo indefinido.

Bibliografía y fuentes

Analizando el Internet de las Cosas. Primera Parte. Disponible en: http://ax3s.blogspot. com.ar/2015/01/ldC09.html Analizando el Internet de las Cosas. Segunda ParteDisponible en: http://ax3s.blogspot.com .ar/2015/01/ldC-10.html

El internet de las cosas, ¿una amenaza para la humanidad? (5 julio de 2016). Disponible en: http://www.expoknews.com/el-internet-de-las-cosas-una-amenaza-para-la-humanidad/

Servicio Geológico de Estados Unidos. Disponible en: https://www.usgs.gov/

Definición de Desarrollo Sostenible, ONU. Disponible en: http://www.un.org/es/ga/presi dent/65/issues/sustdev.shtml

Bureau International des Poids et mesures. Disponible en:http://www.bipm.org/en/ measurementunits/prefixes.html

The Coal Question. An Inquiry Concerning the Progress of the Nation, and the Probable Exhaustion of Our CoalMines (W. Stanley Jevons, 1865). ISBN 978-0-678-00107-3.

Internet de las Cosas. Disponible en: https://es.wikipedia.org/wiki/lnternet_de_las_cosas

Abstract: The term Internet of Things (IoT) has permeated our society in recent years, especially in the media. Although the term is not new, the massification of electronic devices, the real possibility of analyzing large 
amounts of data through Big Data and drawing useful conclusions from them, seems to have definitely opened the door for an loT explosion.

The present article seeks to reflect on a problem that does not seem to have been adequately addressed: its environmental and social implications. In other words, in its sustainability. In a world that is reaching its limits, is it possible that Internet of Things can develop? And if so, will it be for all, helping reduce the gap between those who have the most and those who have the least? Or on the contrary, will these differences increase?

Key words: Internet of Things - loT - Sustainability - Environmental Impacts - Jevons Paradox - Growth Limits.

Resumo: O termo Internet das Coisas (IoT) penetrou na sociedade nos últimos anos de modo especialmente intensa nos meios de comunicação. Embora o termo não é novo, a massificação de dispositivos eletrônicos, a possibilidade real de analisar grandes quantidades de dados através do Big Data e de tirar conclusões úteis deles abriu definitivamente a porta para uma explosão do loT.

Este artigo procura refletir sobre um problema que não parece tratado de modo adequado: suas implicâncias ambientais e sociais; finalmente, em sua sustentabilidade. Num mundo que está alcançando seus limites, é possível que Internet das Coisas possa desenvolver-se? Em fato de fazê-lo, será para todos, ajudando a reduzir o fosso entre os que mais têm e os que menos têm? Ou, pelo contrário, aumentará essas diferenças?

Palavras chave: Internet das Coisas - IoT - sustentabilidade - impactos ambientais - Paradoxo de Jevons - limite do crescimento.

La sustentabilidad de Internet de las Cosas fue publicado de la página 37 a página44 en Cuadernos del Centro de Estudios de Diseño y Comunicación №70 